\title{
Zur Kenntnis des Neurokeratins.
}

\author{
Von \\ Dr. med. Alfred Argiris.
}

(Aus der chemischen Abteilung des physiologisschen Instituts zu Berlin.

(Der Redaktion zugegangen am 15. November 1907.)

Neurokeratin, die Substanz, aus welcher das Sporgiosagerüst der markhaltigen Nervenfasern besteht, wurde von Kühn ${ }^{1}$ ) zuerst dargestellt und analysiert. Kühne teilt auch $\mathrm{mi}$, daß beim Kochen mit Schwefelsäure Tyrosin und Leucin aufreten. Da weitere Angaben über die bei der Hydrolyse entstelenden Substanzen nicht vorliegen, so habe ich, einer Aufforlerung von Herrn Prof. Thierfelder folgend, dahin gehende Jntersuchungen angestellt und berichte zunächst über den Teil, welcher die Hexonbasen, das Tyrosin und das Cystin betrifft. Weitere Mitteilungen über die übrigen Monaminoäuren behalte ich mir vor.

\section{Darstellung des Neurokeratins.}

Nach mannigfachen Vorversuchen bin ich bei $\mathrm{d}$ m $\mathrm{im}$ folgenden $z u$ beschreibenden Verfahren stehen gebliêbëi. Es ist im Prinzip das schon von Kühne und Chittenden benutzte, unterscheidet sich aber von diesem in verschirdenen Einzelheiten und besonders dadurch, daß Trypsinverdauung und Extraktion mit Alkohol und Benzolalkohol wiederholt unl zwar immer mit einander abwechselnd auf die Gehirnmasse angewendet wurden. Es wird dadurch eine vollständigere Entfirnung der Myelinstoffe erreicht, als es bei Kühne und Chittznden der Fall war. Im einzelnen verfuhr ich so: die Gehirne (mensch-

1) Ewald u. Kühne, Verh. d. naturh.-med. Vereins zu Heidelberg, N. F., I., S. 357 (1877) und Kühne u. Ghittenden, Zetschrift f. Biol., Bd. XXVI, S. 291 (1889). 
liche) wurden nach Beseitigung der Häute und des anhaftenden Blutes durch die Fleischhackmaschine zerkleinert, mit Aceton drei- bis viermal behandelt und nun nach Entfernung des Wassers und der Hauptmenge des Cholesterins durch ein feines Haarsieb gerieben. Die auf diese Weise in einen Brei verwandelte Masse wird durch wiederholtes Schütteln mit neuen Äthermengen erschöpft, mit $75^{\%} \%$ igem Alkohol bei $40^{\circ}$ so oft behandelt, bis das Filtrat beim Eindampfen keinen nennenswerten Rückstand hinterließ, und schließlich wiederholt mit einer Mischung gleicher Teile Benzol und Alkohol am Rückflußkühler ausgekocht. Die abfiltrierten Massen wurden in Wasser suspendiert, in flachen Schalen durch Erhitzen auf dem Dampfbade von Benzolresten befreit, darauf in großen Zylindergläsern mit Wasser und soviel Natriumcarbonat, daß der Gehalt etwa $0,5 \%$ betrug, versetzt, und nach Zufügen von Pankreatin zwei Wochen bei einer Temperatur von $39^{\circ}$ gehalten. Während dieser Zeit wurde täglich mehrmals umgerührt. In dem Zylindergefäß befand sich nun ein aus groben Partikeln bestehender Bodensatz, darüber eine feinpulverige Schicht und über dieser eine dunkelbraune, aber ganz klare Lösung. Nun wurde tüchtig umgerührt und, nachdem die gröberen Teilchen sich gesenkt hatten, die Flüssigkeit mit der in ihr suspendierten pulverigen Substanz abgehebert und in ein anderes Zylinderglas übergeführt. Nach erfolgter Senkung heberte ich die überstehende klare Flüssigkeit ab und ersetzte sie durch frische Verdauungslösung. Desgleichen wurde der in dem ersten Zylindergefäß zurückgebliebene aus gröberen Partikeln bestehende Bodensatz mit neuer Verdauungsflüssigkeit übergossen, wieder der Verdauung überlassen und weiter in der eben beschriebenen Weise verfahren, bis schließlich nach nochmaliger Wiederholung der ganzen Operation das ganze Material in den feinpulverigen Zustand umgewandelt war. Nachdem das feine Pulver noch eine Zeitlang der Einwirkung frischer Verdauungsflüssigkeit ausgesetzt worden war, wurde es mittels Heber von der überstehenden klaren Lösung befreit, nach Beseitigung der alkalischen Reaktion durch vorsichtigen Zusatz von Salzsäure mit soviel Alkohol versetzt, daß der Prozentgehalt an Alkohol etwa 75 betrug, und am Rück- 
flußkühler gekocht. Beim Abkühlen des heißen Filtrats schieden sich wieder Myelinstoffe ab, obgleich die Extraktion vor der Verdauung so gut wie beendet war. Eine Erklärung dafür istt wohl in der durch die Verdauung bewirkten feinen Verteilung des Materials zu suchen, vielleicht sind aber im Gehirn auch Myelinstoffeiweißverbindungen enthalten, deren Myelinkomponente erst durch die Verdauung frei und alkohollöslich wird. Es mögen auch beide Momente eine Rolle spielen. An die Alkoholextraktion schloß sich eine solche mit Benzolalkohol an. Jetztt wurde die Masse wieder der tryptischen Verdauung unterworfen und diese und die Extraktion mit den genannten Lösungsmitteln alternierend so oft wiederholt, bis die Verdauungsflüssigkeit keine Biuretreaktion mehr zeigte und die Alkohol- und Benzolalkoholauszüge keine Myelinsubstanzen mehr enthielten. Nunmehr behandelte ich den feinen Brei, welcher sich übrigens ebenso, wie bei allen vorangehenden Filtrationen leicht und ohne Beimengung von Papierfasern vom Filter nehmen ließ, zur Entfernung von anorganischen Bestandteilen mit 0,1\%iger Salzsäure, dann mit Wasser, um die Salzsäure zu beseitigen, und zuletzt mit Alkohol und Äther. In der Reibschale fein zerrieben und durch ein engmaschiges Seidenfilter gesiebt, stellte das Neurokeratin ein hellgelbes, geruchloses Pulver dar.

In Ergänzung der von Kühne gemachten Angaben sei noch erwähnt, daß das Neurokeratin die Farbenreaktionen der Proteine gibt, auch die Probe von Adamkiewicz, nicht aber oder nur sehr schwach die Probe von Molisch.

Der Aschegehalt meiner Präparate betrug im Mittel 0,62 ! ! . Die gefundenen Werte schwankten zwischen 0,61 und $0,64 \%$. Die Kühneschen Präparate enthielten zum Teil etwa die gleiche Menge $(0,74 \%$ ), zum Teil mehr (bis zu 2,4\%). Für die Analysen wurde die Substanz bei $110^{\circ}$ bis zum konstanten Gewicht getrocknet. Den Kohlenstoff und Wasserstoff bestimmte ich nach Dennstedt. Den Stickstoff nach Kjeldahl, den Schwefel nach Asbóth, in der von Neumann und Meinertz ${ }^{1}$ ) angegebenen Modifikation mit folgenden Resultaten:

') Diese Zeitschrift, Bd. XLIII, S. 37. 
1. $0,1306 \mathrm{~g}$ lieferten $0,2695 \mathrm{~g} \mathrm{CO}_{8}$ und $0,0878 \mathrm{~g} \mathrm{H}_{8} \mathrm{O}$ $=56,27 \% \mathrm{C}$ und $7,46 \% \mathrm{H}$.

2. $0,0953 \mathrm{~g}$ lieferten $0,1965 \mathrm{~g} \mathrm{CO}_{2}$ und $0,0631 \mathrm{~g} \mathrm{H}_{2} \mathrm{O}$ $=56,24 \% \mathrm{C}$ und $7,35 \% \mathrm{H}$.

3. $0,3791 \mathrm{~g}$ verbrauchten $38 \mathrm{ccm} \mathrm{n/10-}-\mathrm{H}_{2} \mathrm{SO}_{4}=14,07 \% \mathrm{~N}$

4. 0,3946 , > 39,6 , > $=14,09 \%$,

5. $0,5088 \mathrm{~g}$ lieferten $0,0826 \mathrm{~g} \mathrm{BaSO}_{4}=2,23 \% \mathrm{~S}$

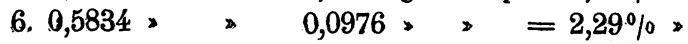

Zusammensetzung der aschefreien Substanz:

$$
\begin{aligned}
& \mathrm{G}=56,62 \%, \quad 56,59 \% \\
& \mathrm{H}=7,51 \%, \quad 7,40 \% \\
& \mathrm{~N}=14,16 \%, \quad 14,17 \% \\
& \mathrm{~S}=2,24 \%, \quad 2,31 \%
\end{aligned}
$$

Die von Kühne und Chittenden dargestellten Neurokeratinpräparate I und II batten einen ganz ähnlichen Gehalt an $\mathrm{C}, \mathrm{H}$ und $\mathrm{N}$ (auf aschefreie Substanz berechnet im Mittel $56,20 \% \mathrm{C}, 7,30 \% \mathrm{H}, 14,18 \% \mathrm{~N}$ ), aber einen geringeren Gehalt an $S(1,75 \%)$. Das hängt vermutlich mit der Einwirkung der Kalilauge zusammen, welche Kühne und Chittenden zur Reinigung benutzten. Die drei anderen von Kühne und Chittenden dargestellten Präparate zeigen eine abweichende $\mathrm{Zu}$ sammensetzung und zwar enthalten sie mehr. Kohlenstoff und weniger Stickstoff.

\section{Hexonbasen.}

$50 \mathrm{~g}$ Neurokeratin wurden mit einer Mischung von $150 \mathrm{~g}$ konzentrierter Schwefelsäure und $300 \mathrm{~g}$. Wasser am Rückflußkühler gekocht, und zwar in einem geräumigen Kolben, da die Flüssigkeit im Anfang sehr stark schäumt. Während des Erhitzens entweicht Schwefelwasserstoff, daneben entwickelt sich eine andere durch einen unangenehmen, nicht genauer zu definierenden Geruch ausgezeichnete flüchtige Substanz, deren Natur nicht festgestellt werden konnte. Methylmerkaptan und Äthylsulfid ließen sich nicht nachweisen. Nach 24 stündigem Kochen wurde die stark braune Flüssigkeit, welche keine Biuretreaktion mehr zeigte, von einem ungelösten Rückstand abfiltriert, der Rückstand mit kochendem Wasser bis zum Aufhören der Schwefelsäurereaktion im Filtrat ausgewaschen, bei $100^{\circ}$ getrocknet und gewogen. Seine Menge betrug $7,5 \mathrm{~g}=15 \%$ des 
angewendeten Neurokeratins. Er enthielt 0,68 g (=9\%) Stickstoff, so daß also etwa 10\% des Gesamtstickstoffs des Neurokeratins nicht in Lösung gegangen ist. Die Menge des Rückstandes ist auffallend hoch, weit höher als z. B. bei Roßhaaren, von welchen bei gleicher Behandlung nur etwa $4 \%$ nicht in Lösung gingen (siehe S. 92).

Daß das Maximum der Spaltung, welches Schwefelsäure der benutzten Konzentration $\mathrm{zu}$ bewirken vermag, erreicht worden ist, ergibt sich aus einem zweiten Versuch, in dem bei nur 18 stündigem Kochen fast die gleichen Verhältnisse gefunden wurden. Der ausgewaschene und getrocknete Rückstand wog. in diesem Fall 7,8 g. Seine Menge betrug also 15,6\% des Neurokeratins. Er enthielt $0,67 \mathrm{~g}\left(=8,6^{\circ} / \mathrm{n}\right)$ Stickstoff.

Von diesem gegen Schwefelsäure widerstandsfähigen Rückstand wird aber noch ein erheblicher Teil beim Erhitzen mit Salzsäure gelöst, wie sich aus folgendem Versuch ergibt. $15,3 \mathrm{~g}$ der trockenen Masse wurden 6 Stunden mit konzentrierter Salzsäure gekocht; der abfiltrierte ausgewaschene und getrocknete Rückstand wog jetzt $9,9 \mathrm{~g}$ und enthielt $0,72 \mathrm{~g}$ Stickstoff. Es war also noch ungefähr ein Drittel der Substanz. und die Hälfte des Stickstoffs in Lösung gegangen. Das Filtrat wurde nach bekanntem Verfahren auf Hexonbasen untersucht. Sie waren vermutlich vorhanden, da an den betreffenden Stellen Niederschläge entstanden, aber in sehr kleinen Mengen, so daß eine Gewinnung in krystallisierter Form nicht gelang. In dem Quecksilbersulfatniederschlag $z$. B., welcher das Histidin enthalten mußte, fand sich nur $0,008 \mathrm{~g} \mathrm{~N}$, entsprechend $0,029 \mathrm{~g}$ Histidin.

Filtrat und Waschwasser wurden vereinigt und mit so viel Baryumhydroxyd versetzt, daß die vom Baryumsulfatniederschlag abfiltrierte Flüssigkeit ( + Waschwasser) nach dem Einengen auf $500 \mathrm{ccm}$ noch $5 \%$ freie Schwefelsäure enthielt. $\mathrm{Zu}$ der klaren Lösung fügte ich solange 30\% ige Phosphorwolframsäure, bis eine abfiltrierte Probe nach weiterem Zusatz des Fällungsmittels innerhalb 10 Minuten klar blieb, isolierte den Niederschlag durch starkes Absaugen und Pressen und verrieb ihn mit Wasser. Er wurde nun abermals abgesaugt und abgepreßt, dann in Wasser suspendiert, mit heißgesättigter Barytlösung zerlegt, die abfiltrierte Flüssigkeit nach Entfernung des; überschüssigen Baryums mit Schwefelsäure angesäuert. Durch sorgfältiges Auswaschen wurde dafür gesorgt, daß alle diese: 
Operationen ohne Verlust verliefen. Die weitere Isolierung und quantitative Bestimmung des Histidins, Arginins und Lysins geschah nach den von Kossel, Kutscher und Patten ${ }^{1}$ ) gegebenen Vorschriften. Dabei machte ich die Beobachtung, daß gleich im Anfang des Verfahrens beim Versetzen der schwefelsauren Lösung mit Silbernitrat ein weißer flockiger Niederschlag entstand. Ich filtrierte ihn ab, suspendierte ihn in schwefelsäurehaltigem Wasser, zersetzte ihn mit.Schwefelwasserstoff, filtrierte vom Schwefelsilber ab, entfernte die Schwefelsäure mit Baryumcarbonat und dampfte ein. Der zurückbleibende Syrup reagierte stark alkalisch, die Menge war aber so gering, daß von einer weiteren Untersuchung abgesehen werden mußte. Ein gleicher Niederschlag trat an der gleichen Stelle bei der Untersuchung der. hydrolytischen Spaltungsprodukte der Roßhaare auf. Es handelte sich vielleicht um die Baryumverbindung einer Aminosäure.

Histidin. Die Versuche, das aus dem Quecksilbersulfatniederschlage isolierte Histidin als Histidindichlorid zur Krystallisation zu bringen, schlugen fehl. Ich habe mich deswegen damit begnügen müssen, in der Lösung, welche $100 \mathrm{ccm}$ betrug, den Stickstoff $z u$ bestimmen, und aus der Menge des Stickstoffs die Menge des Histidins zu berechnen. Es wurden in zwei übereinstimmenden Versuchen in je $15 \mathrm{ccm} 15,58 \mathrm{mg} \mathrm{N}$ gefunden. Das entspricht 0,760\% Histidin.

Arginin. Es wurde als Kupfernitratverbindung isoliert und gewogen. Die Menge betrug 1,94 $\mathrm{g}=3,88 \%=2,28 \%$ Arginin. Die Reinheit des Salzes geht aus dem Schmelzpunkt, welcher bei $114^{\circ} \mathrm{lag}$, und aus der Analyse hervor.

$0,1818 \mathrm{~g}$ lieferten $0,1644 \mathrm{~g} \mathrm{CO}_{2}$ und $0,0958 \mathrm{~g} \mathrm{H}_{\mathbf{2}} \mathrm{O}$ $=24,64 \% \mathrm{C}$ und $5,82 \% \mathrm{H}$.

$0,1314 \mathrm{~g}$ lieferten 0,0178 g $\mathrm{CuO}=10,81 \% \mathrm{Cu}$.

Gefunden: $\quad$ Berechnet für $2 \mathrm{C}_{6} \mathrm{H}_{14} \mathrm{~N}_{4} \mathrm{O}_{2} \cdot \mathrm{Cu}\left(\mathrm{NO}_{8}\right)_{2}+3 \mathrm{H}_{8} \mathrm{O}$ :

$$
\begin{array}{lr}
\mathrm{C}=24,64 \% & 24,40 \% \\
\mathrm{H}=5,82 \% & 5,78 \% \\
\mathrm{Cu}=10,81 \% & 10,79 \%
\end{array}
$$

Lysin. Es wurde als Lysinpikrat abgeschieden und gewogen. Seine Menge betrug $3,52 \mathrm{~g}=7,04 \%=2,72 \%$ Lysin.

1) Diese Zeitschrift, Bd. XXXI, S. 165, und Bd. XXXVIII, S. 39. 
Für die Reinheit spricht das Ergebnis der Analyse.

1. $0,1500 \mathrm{~g}$. lieferten $0,2130 \mathrm{~g} \mathrm{CO}_{2}$ und $0,0654 \mathrm{~g} \mathrm{H}_{2} \mathrm{O}$ $=38,66 \% \mathrm{G}$ und $4,80 \% \mathrm{H}$.

2. $0,2080 \mathrm{~g}$ lieferten $0,2934 \mathrm{~g} \mathrm{CO}_{2}$ und $0,1000 \mathrm{~g} \mathrm{H}_{2} \mathrm{O}$ $=38,46 \% \mathrm{C}$ und $5,31 \% \mathrm{H}$.

$$
\begin{array}{ccc}
\multicolumn{2}{c}{\text { Gefunden: }} & \text { Berechnet für } \mathrm{C}_{86} \mathrm{H}_{14} \mathrm{~N}_{2} \mathrm{O}_{2} \cdot \mathrm{C}_{8} \mathrm{H}_{2}\left(\mathrm{NO}_{2}\right)_{3} \mathrm{OH}: \\
\mathrm{C}=38,66 \% & 38,46 \% & 38,40 \% \\
\mathrm{H}=4,80 \% & 5,31 \% & 4,53 \%
\end{array}
$$

In dem oben schon erwähnten zweiten Spaltungsversuch, bei dem nur 18 Stunden gekocht wurde, der aber im übrigen in derselben Weise ausgeführt wurde, erhielt ich ganz übereinstimmende Resultate. Die Menge des Arginins betrug 2,19\%, die des Lysins 2,68\%, Histidin konnte auch hier nicht als salzsaures Salz krystallisiert gewonnen werden.

Von andern, in die Gruppe der Keratine gehörigen Substanzen ist nur die Hornsubstanz in bezug auf ihren Hexonbasengehalt untersucht worden. Hedin ${ }^{1}$ ) wies unter den Zersetzungsprodukten des Hornes Lysin und Arginin nach und gibt die Menge des letzteren $\mathrm{zu}$ mindestens 2,25\% an. Abderhalden und Voitinovici²) fanden im Horn (von Hammeln) 2,7\% Arginin und 0,2\% Lysin, während sie Histidin nicht isolieren konnten.

Ich habe das oben beschriebene Untersuchungsverfahren auch auf gut ausgewaschene und mit Alkohol und Äther behandelte Roßhaare (40 g) angewendet und folgende Resultate erhalten.

Der bei der Hydrolyse ungelöst zurückbleibende Teil betrug, wie oben schon erwähnt, $1,57 \mathrm{~g}=3,9 \%$ und enthielt $0,11 \mathrm{~g}$ Stickstoff. Histidin konnte nicht als krystallisiertes Chlorid erhalten werden. Aus dem Stickstoffgehalt des Quecksilbersulfatniederschlags berechnet, betrug seine Menge 0,61\%. Das Argininkupfernitrat (Schp. $115^{\circ}, \mathrm{Cu}$ gef. 10,66, ber. 10,79) wog 3,02 g. Das entspricht $7,55 \%$ oder $4,45 \%$ Arginin. Die Menge des Lysinpikrats (gef. 38,25\% $\mathrm{C}$ und $4,83 \% \mathrm{H}$, ber. $38,40 \% \mathrm{C}$ und $4,53 \% \mathrm{H}$ ) betrug $1,08 \mathrm{~g}=2,70 \%=1,12 \% \mathrm{~L} \mathrm{y} \sin$.

1) Lund's Univers. Arsskrift, T. XXIX. - Diese Zeitschrift, Bd. XXI, S. 155 .

2) Diese Zeitschrift, Bd. LII, S. 348. 


\section{Tyrosin.}

$50 \mathrm{~g}$ Neurokeratin wurden mit $25 \%$ iger Schwefelsäure 18 Stunden am Rückflußkühler gekocht. Nach Filtration und Auswaschen des Rückstandes mit heißem Wasser wurden die gesamten Filtrate durch Baryumbydroxyd von der Schwefelsäure und durch Kohlensäure vom überschüssigen Baryum befreit und die Niederschläge wiederholt mit Wasser ausgekocht. Das beim Einengen der vereinigten Filtrate sich abscheidende Baryumcarbonat filtrierte ich $a b$ und dampfte dann soweit ein, bis sich bereits in der. Wärme eine Krystallhant bildete: Nach dem Abkühlen warde sie abfiltriert und das Filtrat weiter eingeengt zur Gewinnung weiterer Krystalle. Die Menge des Tyrosins betrug nach Entfärbung mit Tierkohle und Umkrystallisation $2,3 \mathrm{~g}=4,6 \%$.

$0,1042 \mathrm{~g}$ lieferten $0,2299 \mathrm{~g} \mathrm{CO}_{\mathrm{z}}$ und $0,0590 \cdot \mathrm{g} \mathrm{H}_{8} \mathrm{O}$ $=59 ; 6 \mathrm{1} \% \mathrm{C}$ und $6 ; 23 \% \mathrm{H}$,

während die Formel $\mathrm{C}_{8} \mathrm{H}_{11} \mathrm{NO}_{8} 59,66 \% \mathrm{G}$ und 6,07\% $\mathrm{H}$ verlangt.

Cystin.

Es wurden $10 \mathrm{~g}$ Neurokeratin 6 Stunden mit konzentrierter Salzsäure am Rückflußkühler gekocht. Der abfiltrierte Rückstand wog nach Auswaschen und Trocknen 0,828 $\mathrm{g}$ und enthielt $0,0215 \mathrm{~g} \mathrm{~S}$ (zwei gut miteinander übereinstimmende Analysen). Es waren also 91,7\% der Substanz und fast 91\% des Schwefels in Lösung gegangen.

Die Gewinnung des Cystins aus dieser hydrolytischen Flüssigkeit machte mir große Schwierigkeiten, obgleich mit dem von mir eingeschlagenen Verfahren die Isolierung aus Hornsubstanz mit Leichtigkeit gelang. Erst als ich in der jetzt zu beschreibenden Weise vorging, war das Resultat ein positives. ${ }^{1}$ ) Ich dampfte Filtrat und Waschwasser bei $40^{\circ}$ im Vakuum ein,

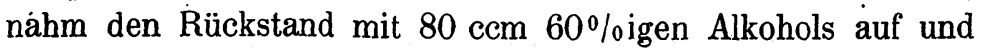
machte die Reaktion durch Hinzufügen von starker (33\% iger) Natronlauge unter starker Abkühlung ganz schwach sauer. Der während dreitägigen Stehens im Eisschrank abgeschiedene

1) Mit diesem Verfahren konnte ich auch aus $15 \mathrm{~g}$ Casein sechsseitige Tafeln von Cystin gewinnen. 
Niederschlag wurde mit wenig heißem Wasser aufgenommen und durch Hinzufügung von möglichst wenig Salzsäure in Lösung gebracht. Aus der Lösung schied sich nach Entfärben mit Tierkohle und Einengen beim langsamen Abkühlen Cystin in Form sechsseitiger Tafeln ab. Die Menge betrug 0,0340 g. Die Mutterlauge wurde mit essigsaurem Natron versetzt, bis sich Kongopapier nicht mehr blau färbte, und eingeengt. Es schieden sich weitere Fraktionen ab, welche aber nicht von dem gleichzeitig sich abscheidenden Tyrosin getrennt werden konnten. Ich habe deshalb diese Fraktionen gelöst, die Lösung auf $100 \mathrm{ccm}$ gebracht und in je $25 \mathrm{ccm}$ eine Schwefelbestimmung ausgeführt. Es wurden erhalten 0,0568 und $0,0562 \mathrm{~g}$, im Mittel $0,0565 \mathrm{~g}$ $\mathrm{BaSO}_{4}=0,0078 \mathrm{~g} \mathrm{~S}$. In $100 \mathrm{ccm}$ waren also $0,0312 \mathrm{~g} \mathrm{~S}$ oder $0,116 \mathrm{~g}$ Cystin enthalten. Die Gesamtmenge des Cystins betrug also $0,150 \mathrm{~g}=1,50 \%$ Cystin. Die Mutterlaugen gaben noch starke Schwefelbleireaktion, doch war es nicht möglich, noch weitere Krystallisationen $\mathrm{zu}$ erhalten.

Es wurden also gewonnen in Prozenten aus:

\begin{tabular}{lccc} 
& \multicolumn{2}{c}{ Neurokeratin } & Ro Bhaaren \\
Lysin & 2,72 & 2,68 & 1,12 \\
Arginin & 2,28 & 2,19 & 4,45 \\
Histidin & 0,76 & & 0,61 \\
Tyrosin & 4,60 & & \\
Cystin & 1,50 & &
\end{tabular}

